A. Pramesh Rao, G. Swarup and Gopal-Krishna, eds.

\title{
A Large Diffuse Radio Source in a Cluster of Galaxies at $z=0.13$
}

\author{
Gopal-Krishna $^{1}$, V.K. Kulkarni ${ }^{1}$, J. Bagchi ${ }^{2}$ and J. Melnick ${ }^{3}$ \\ ${ }^{1}$ NCRA-TIFR, Pune University Campus, Pune 411007, India. \\ ${ }^{2}$ IUCAA, Pune University Campus, Pune 411007, India. \\ ${ }^{3}$ ESO, Casilla 19, Santiago, Chile
}

\begin{abstract}
Discovery of a diffuse ultra-steep spectrum radio source of size $\sim 0.3 \mathrm{Mpc}$, possibly a radio halo, in a cluster of galaxies at $z=0.13$ is reported hilighting the presence of a giant radio spiral within the halo.
\end{abstract}

We present GMRT and VLA radio maps and NTT optical image/spectrum of an ultra-steep-spectrum $(\alpha \sim-1.3)$, highly diffuse radio source $0116+111$ found in the Ooty Lunar Occultation Survey (Joshi \& Singal, 1980). Its spectrum is derived from measured flux densities of $920 \pm 24 m J y$ at $327 \mathrm{MHz}$ (Joshi \& Singal, 1980), $810 \pm 60 \mathrm{~mJ} y$ at $408 \mathrm{MHz}$ (Large et al, 1981), 448 $\pm 12 m J y$ at 610 $\mathrm{MHz}$ (present work), $147 \pm 8 m J y$ at $1.4 \mathrm{GHz}$ (Condon et al, 1998), $60 \pm 9 \mathrm{~mJ} y$ at $2.7 \mathrm{GHz}$ (Effelsberg telescope, present work), $30 \pm 5 \mathrm{mJy}$ at $4.9 \mathrm{GHz}$ (Gregory et al, 1996) and $35 \pm 4 m J y$ at $4.9 \mathrm{GHz}$ (present work). Earlier, our C-array VLA map revealed an amorphous radio emission $\left(\sim 1^{\prime}\right.$ at $\left.5 \mathrm{GHz}\right)$, without any unresolved component above $1 \mathrm{mJy}$. Based on a R-band NTT image (Fig. 1), the source is identified with $\mathrm{a} \sim 17-\operatorname{mag} \mathrm{cD}$ galaxy $\left(\alpha_{1950}=011623.52, \delta_{1950}\right.$ $=+110735.0$ ), which appears to be the dominant member of a distant cluster. A slit-spectrum taken with the grism-3 optics of the NTT gave a redshift $z=$ 0.1316 , based on the absoption lines of $\mathrm{Na}(\lambda 5893), \mathrm{Mgb}(\lambda 5169), \mathrm{H} \beta$, G-band $(\lambda 4304)$, the H,K break and a probable [O II] $\lambda 3727$ emission line. Also, the bright elliptical $\sim 15^{\prime \prime}$ south of the $\mathrm{cD}$ is found to have $z=0.1309$.

The VLA maps (Figs. 2 \& 3) show two warm spots straddling the $\mathrm{cD}$ along $\mathrm{PA} \sim 50^{\circ}$, indicating a jet-like outflow from the $\mathrm{cD}$. The amorphous radio structure underlying these peaks has an overall extent of $2^{\prime}$ in the GMRT map at $610 \mathrm{MHz}$ (i.e., $\sim 0.3 \mathrm{Mpc}$,for $\mathrm{H}_{o}=65 \mathrm{Kms}^{-1} . \mathrm{Mpc}^{-1}$; Fig. 4). Much of this diffuse radio emission lies to the noth-west of the $\mathrm{cD}$ and has no detected optical counter parts (See Fig. 1). Thus, it probably represents parts of a radio halo associated with this distant cluster. If so, its luminosity $\left(\sim 1.10^{25} \mathrm{~W} . \mathrm{Hz}^{-1}\right.$ at $610 \mathrm{MHz}$ ) would place it among the most luminous radio halos known.

From Figs. 2-4, it is seen that the orientation of the two radio peaks undergoes a systematic clockwise progression with frequency. In fact, the two peaks appear to be part of a radio ridge emanating form the $\mathrm{cD}$ and extending well beyond the two peaks, taking the form of a huge radio 'barred spiral' with a diameter of $\sim 100 \mathrm{kpc}$ (Fig. 2b). Quite plausibly, such an edge-darkened morphology of radio jets facilitates leakage of their relativistic particles which can fill the giant radio halo. Further in-situ particle acceleration within the halo could occur in the turbulent wakes of the cluster galaxies (Jaffe 1977) and/or in the shocks caused by merger of sub-clusters (De Young, 1992; Tribble, 1993). 

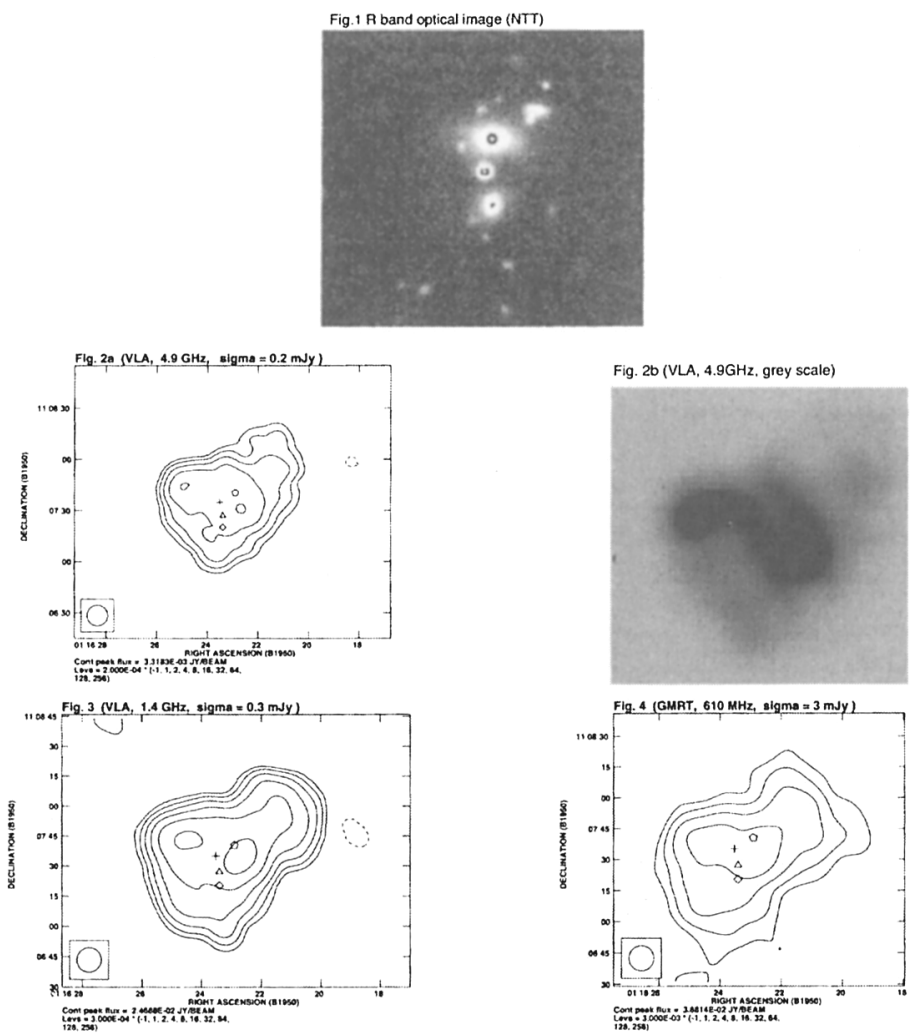

Acknowledgments. The VLA is operated by the National Radio Astronomy Observatory (NRAO) for Associated Universities Inc. under a licence from the National Science Foundation of the USA.

\section{References}

Condon, J. J. et. al. 1998, AJ 115, 1693

De Young, D. S., 1992, ApJ 386, 464

Ekers, R. D., Fanti, R., Lari, C. \& Parma, P., 1978, Nature 276, 588

Gopal-Krishna \& Steppe, H., 1982. A\&A 113, 150

Gregory, P. C., Scott, W. K., Douglas, K. \& Condon, J. J., 1996, ApJS 103427 Jaffe, W. J., 1977, ApJ 212, 1

Joshi, M. N. \& Singal, A. K., 1980, Mem. Astr. Soc. India, 1,49

Large, M. I., Mills, B. Y., Little, A. G., Crawford, D. F. \& Sutton, J. M., 1981, MNRAS 194, 693

Tribble, P. C., 1993, MNRAS 263, 31 